\title{
Dendritic Spines Lost during Glutamate Receptor Activation Reemerge at Original Sites of Synaptic Contact
}

\author{
M. Josh Hasbani, Michelle L. Schlief, Daniel A. Fisher, and Mark P. Goldberg \\ Departments of Neurology and Anatomy and Neurobiology Center for the Study of Nervous System Injury, Washington \\ University School of Medicine, St. Louis, Missouri 63110
}

During cerebral ischemia, neurons undergo rapid alterations in dendritic structure consisting of focal swelling and spine loss. We used time-lapse microscopy to determine the fate of dendritic spines that disappeared after brief, sublethal hypoxic or excitotoxic exposures. Dendrite and spine morphology were assessed in cultured cortical neurons expressing yellow fluorescent protein or labeled with the fluorescent membrane tracer, Dil. Neurons exposed to NMDA, kainate, or oxygenglucose deprivation underwent segmental dendritic beading and loss of approximately one-half of dendritic spines. Most spine loss was observed in regions of local dendritic swelling. Despite widespread loss, spines recovered within $2 \mathrm{hr}$ after termination of agonist exposure or oxygen-glucose deprivation and remained stable over the subsequent $24 \mathrm{hr}$. Recovery was slower after NMDA than AMPA/kainate receptor activation. Time-lapse fluorescence imaging showed that the vast majority

The dendritic spine is a basic functional unit of integration of neuronal circuits and a site of structural and functional synaptic plasticity. Spines are subject to early and selective damage during cerebral ischemia. Within minutes of interruption of cerebral blood flow, there is the appearance of focal dendritic swelling and the disappearance of dendritic spines (Ramon y Cajal, 1909, 1995; Ikonomidou et al., 1989; Hsu and Buzsaki, 1993; Matesic and Lin, 1994). A similar pattern of hypoxic injury is observed in slice preparations and cell culture models (Stewart et al., 1991; Hori and Carpenter, 1994; Park et al., 1996; Jarvis et al., 1999). There is abundant evidence in vivo and in vitro that these rapid structural changes are caused by activation of excitatory amino acid pathways. More than $90 \%$ of dendritic spines in the mammalian CNS are contacted by excitatory synapses (Harris and Kater, 1994), rendering these postsynaptic structures vulnerable to conditions of excessive glutamate release. Hypoxic spine loss in vivo and in culture can be reproduced by direct application of glutamate agonists (Olney, 1971; Olney et al., 1979; Park et al., 1996; Halpain et al., 1998) and prevented by glutamate receptor blockade (Park et al., 1996). Hypoxic and excitotoxic alterations

\footnotetext{
Received Aug. 22, 2000; revised Jan. 16, 2001; accepted Jan. 21, 2001.

This work was supported by National Institutes of Health Grants NS32636 and NS40138. This work was performed during the tenure of an Established Investigator Award from the American Heart Association. We thank William D. Snider for assistance with transfection protocols and Krzysztof L. Hyrc for performing calcium imaging. Kelvin A. Yamada, Ann Marie Craig, and Steven M. Rothman provided helpful discussion and review of this manuscript. We thank Olga Strots for expert technical assistance.

Correspondence should be addressed to Dr. Mark P. Goldberg, Department of Neurology, Campus Box 8111, Washington University School of Medicine, $660 \mathrm{~S}$. Euclid Avenue, St. Louis, MO 63110. E-mail: goldberg@neuro.wustl.edu. Copyright (C) 2001 Society for Neuroscience $0270-6474 / 01 / 212393-11 \$ 15.00 / 0$
}

of spines reemerged in the same location from which they disappeared. In addition to spine recovery, elaboration of dendritic filopodia was observed in new locations along the dendritic shaft after dendrite recovery. Spine recovery did not depend on actin polymerization because it was not blocked by application of latrunculin-A, which eliminated filamentous actin staining in spines and blocked spine motility. Throughout spine loss and recovery, presynaptic and postsynaptic elements remained in physical proximity. These results suggest that elimination of dendritic spines is not necessarily associated with loss of synaptic contacts. Rapid reestablishment of dendritic spine synapses in surviving neurons may be a substrate for functional recovery after transient cerebral ischemia.

Key words: hypoxia; glutamate; excitotoxicity; dendritic spine; synapse; actin

in synaptic elements may contribute to rapid disruption of neurological function occurring within minutes of energy depletion in the brain.

Little is known about the fate of dendritic spines in neurons that survive acute neurological insults. Delayed restoration of spine density has been proposed to contribute to functional improvement in experimental models of cortical aspiration lesions (Kolb and Gibb, 1993; Rowntree and Kolb, 1997) and global ischemia (Akulinin et al., 1997). Recovery in spine density has also been observed in models of experimental epilepsy (Muller et al., 1993; Isokawa, 1998). However, it is not known whether the observed changes in apparent spine density in neuronal populations are caused by selective loss of degenerating cells or by actual spine recovery. Furthermore, such studies rely on conventional histological measures, which do not demonstrate dynamic changes in individual dendritic spines. If spines recover after loss, do they emerge in former or new locations? Are synaptic contacts lost when spines disappear, and if so, do spines that recover reassociate with presynaptic terminals? To address these questions, we examined spine loss and recovery after hypoxia or glutamate receptor activation using time-lapse confocal microscopy in cultured cortical neurons. The defined architecture and accessibility of primary dissociated culture allowed highresolution visualization of dendritic spines and presynaptic elements in neurons visualized by expression of yellow fluorescent protein (YFP), a green fluorescent protein derivative, or labeled by application of the fluorescent membrane tracer, DiI.

\section{MATERIALS AND METHODS}

Mouse cortical cell culture. Neocortices from day 15 murine embryos were dissociated and plated on confluent astrocyte cultures at 1 week in vitro 
as described previously (Rose et al., 1993). Briefly, neurons were plated at a density of three neocortex hemispheres per $10 \mathrm{ml}$ plating media that contained 5\% horse serum, 5\% fetal bovine serum, 2 mm glutamine, 26.2 $\mathrm{mM} \mathrm{NaHCO}_{3}$, and $20 \mathrm{~mm}$ D-glucose in MEM. Cultures were maintained at $37^{\circ} \mathrm{C}$ with $5 \% \mathrm{CO}_{2}$. Most of the neurons are glutamatergic $(\sim 90 \%)$, with a small proportion that contain GABA or other neurotransmitters (Yin et al., 1994). Experimental procedures were conducted on cultures at $14-17 \mathrm{~d}$ in vitro, when an excitotoxic response could be elicited.

Excitatory amino acid exposure and oxygen-glucose deprivation. Cultures were exposed to $30 \mu \mathrm{M}$ NMDA (Sigma, St. Louis, MO) or 100-300 $\mu \mathrm{M}$ kainate (Sigma) for $10 \mathrm{~min}$ in a HEPES- and bicarbonate-buffered balanced salt solution (Hasbani et al., 1998). In some experiments, MK-801 (RBI, Natick, MA) or NBQX (Parke-Davis, Ann Arbor, MI) was included in the recovery buffer. Oxygen-glucose deprivation was performed as described (Goldberg and Choi, 1993; Goldberg et al., 1997). Briefly, cultures were transferred to an anaerobic chamber (Forma Scientific, Marietta, $\mathrm{OH}$ ) containing $5 \% \mathrm{CO}_{2}, 10 \% \mathrm{H}_{2}, 85 \% \mathrm{~N}_{2}$. Culture medium was replaced with deoxygenated, glucose-free balanced salt solution. Exposures were terminated by returning cultures to normal oxygenated medium or by fixation in $4 \%$ paraformaldehyde and $0.025 \%$ glutaraldehyde in PBS at the appropriate time points. These exposure conditions resulted in $<20 \%$ neuronal death (Park et al., 1996), assessed by propidium iodide exclusion $1 \mathrm{~d}$ after exposure (data not shown). Cytosolic calcium elevation after NMDA exposure was determined by the fluorescent indicator fura-2 as described previously (Hasbani et al., 1998).

Green fluorescent protein transfection. Neurons were transfected at 2-3 $\mathrm{d}$ in culture with the plasmid eYFPN1 (ClonTech, Palo Alto, CA), using the DOSPER Liposomal Transfection Reagent (Boehringer Mannheim, Indianapolis, IN) at a ratio of 1:4 plasmid/DOSPER and $0.5 \mu \mathrm{g}$ plasmid per tissue culture well. These conditions were selected to yield a transfection efficiency of $<0.01 \%$, permitting the study of individual neurons (approximately one per $200 \times$ field). Neuronal cell bodies expressed green fluorescent protein (GFP) the day after transfection, and neurites developed over subsequent days. GFP fluorescence was stable in a number of neurons for at least 3 weeks and revealed the neuronal arbor, including axons, dendrites, and dendritic spines.

DiI labeling. Neurons were labeled with the carbanocyanine membrane tracer DiI $\left(\mathrm{C}_{18}\right)_{3}$ ("DiI," Molecular Probes, Eugene, OR) as described previously (Honig and Hume, 1986; Park et al., 1996). In other experiments, a saturated stock of DiI was made in cod liver oil, and individual cells were labeled by micropipette (Papa et al., 1995).
Immunocytochemistry. Fixed cultures were incubated in $0.25 \%$ Triton $\mathrm{X}-100$ at room temperature for $10 \mathrm{~min}$ and blocked in $10 \%$ normal goat serum for $60 \mathrm{~min}$. Antibodies to synapsin (Affinity Bioreagents, Golden, CO; rabbit, 1:250), synaptophysin (Dako, Carpinteria, CA; rabbit, 1:50), or YFP (Chemicon, Temecula, CA; rabbit or chicken, 1:500) were applied for $2 \mathrm{hr}$ at room temperature, followed by appropriate fluorescent Alexa-488- or Alexa-568-conjugated goat anti-rabbit or chicken secondary antibodies (Molecular Probes). Control experiments using single fluorophores demonstrated complete separation of Alexa-488 and Alexa-568 emission.

Microscopy and image acquisition. Low-magnification images (see Figs. $1,2)$ were captured using conventional fluorescence microscopy and a digital camera (Spot; Diagnostic Instruments). Confocal imaging was performed with a laser scanning confocal microscope (Odyssey; Noran Instruments) using a $100 \times$ oil-immersion objective (numeric aperture, 1.4; Nikon) and either $488 \mathrm{~nm}$ excitation and $>515$ emission or $568 \mathrm{~nm}$ excitation and $>590$ emission. A secondary dichroic filter at $560 \mathrm{~nm}$ was used to separate fluorophores for double-labeling experiments (see Fig. 9). Confocal images were acquired with a pixel size of $0.11 \mu \mathrm{m}(512 \times$ 480 pixels). Serial optical sections were obtained at $0.4-0.8 \mu \mathrm{m}$ intervals through the dendritic arbor. Each optical section required $1 \mathrm{sec}$ of scan time and typical stacks consisted of 5-15 optical sections. For time-lapse experiments, laser intensity, gain, offset, and contrast settings were chosen to optimize visualization of spines and filopodia before data acquisition and were not subsequently altered within individual experiments. Images were captured and analyzed with a PC-based system (MetaMorph; Universal Imaging, West Chester, PA).

Analysis of spine and dendrite morphology. The presence of dendritic varicosities in DiI-labeled or YFP-expressing neurons was determined at $400 \times$ under epifluorescence illumination as described previously (Hasbani et al., 1998). For each neuron, varicosities were scored as present if found in at least one dendrite.

Spine protrusions were classified using criteria modified from $\mathrm{Ziv}$ and Smith (1996): spines (with distinct heads) or filopodia (length $>4 \mu \mathrm{m}$ in the $x-y$ plane and lacking heads). Protrusions were scored as spines if the distal end was $>0.22 \mu \mathrm{m}$ (two pixels) wider than the shaft. Protrusion density measurements were determined by acquisition of threedimensional confocal image stacks of 30-100 $\mu \mathrm{m}$ segments of secondary dendrites from each neuron. For quantitative studies of DiI-labeled neurons fixed at various time points, we summed all protrusions, regardless of morphological classification. Because spines changed shape during excitotoxin exposure, the goal was to ensure that alterations in protrusion
Figure 1. YFP expression in primary cortical culture. Cortical neuronal cultures were transfected with YFP at day 2 and examined at day 15 in vitro. Phase-contrast microscopy shows only a phase-bright neuronal cell body ( $A$, arrow), whereas YFP fluorescence in the same field (with identical magnification) reveals the entire dendrite arbor and axon of a single transfected neuron $(B)$. $C$, YFP expression does not alter spine density. YFPtransfected cells, nontransfected cells in the same culture, and sister cultures that never underwent transfection were assessed for protrusion density (number of protrusions/10 $\mu \mathrm{m}$ dendrite length) on day 14 in culture. Nontransfected cells were assessed for spine density by DiI labeling, whereas transfected cells were assessed by YFP expression $(n=10$ neurons from two different culture platings per condition). $D$, YFP expression does not alter NMDA receptor function. Cortical cultures were loaded with Fura-2 AM and exposed to $30 \mu \mathrm{M}$ NMDA for $10 \mathrm{~min}$. Cytosolic calcium elevation $(340 \mathrm{~nm} / 380 \mathrm{~nm}$ excitation ratio) in YFP-transfected cells (YFP+) was similar to nontransfected cells in the same culture (YFP-) and to neurons in sister cultures that did not undergo transfection. Resting calcium ratio data (data not shown) were the same in all groups. Scale bar, $200 \mu \mathrm{m}$.
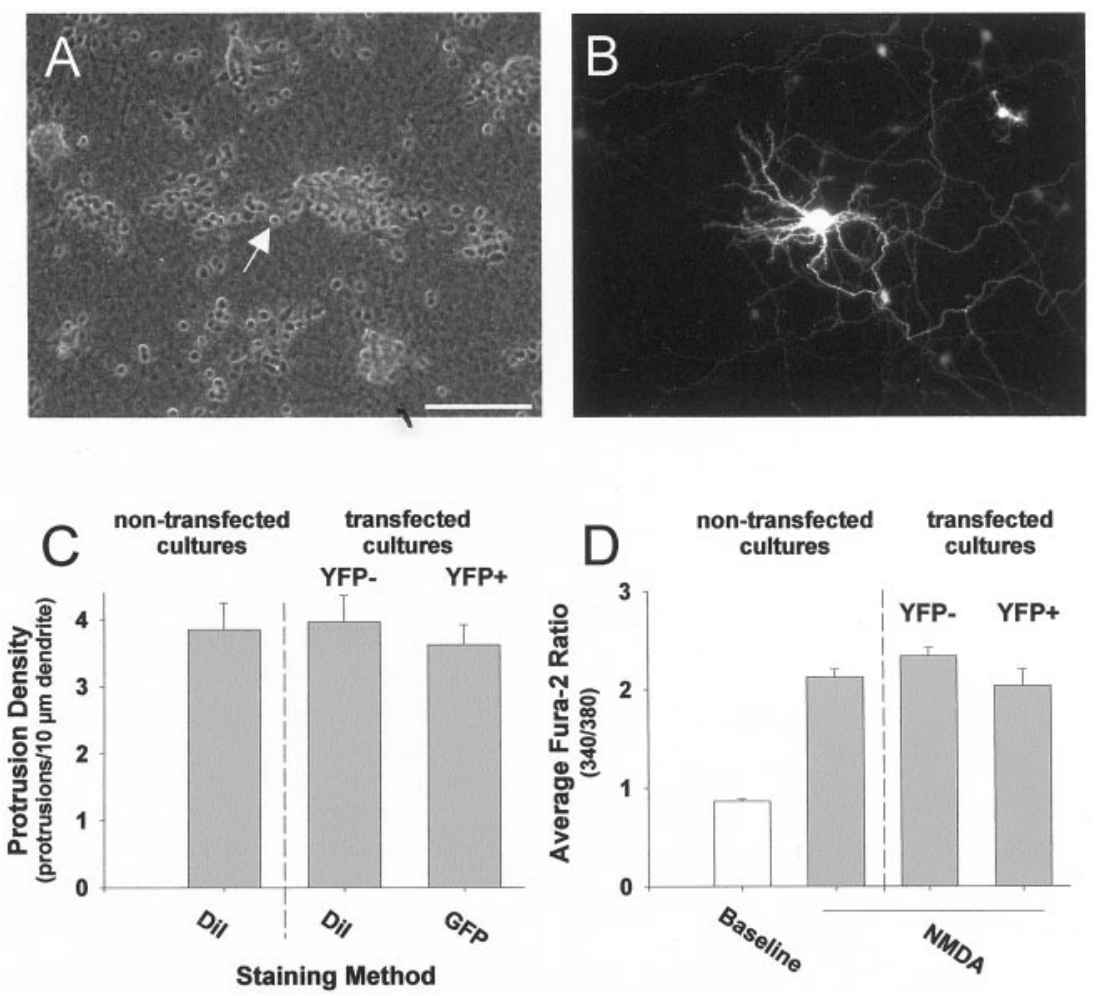

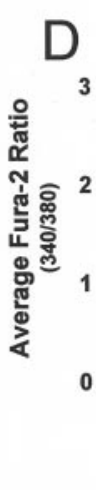

non-transfected

cultures

transfected cultures
YFP. 

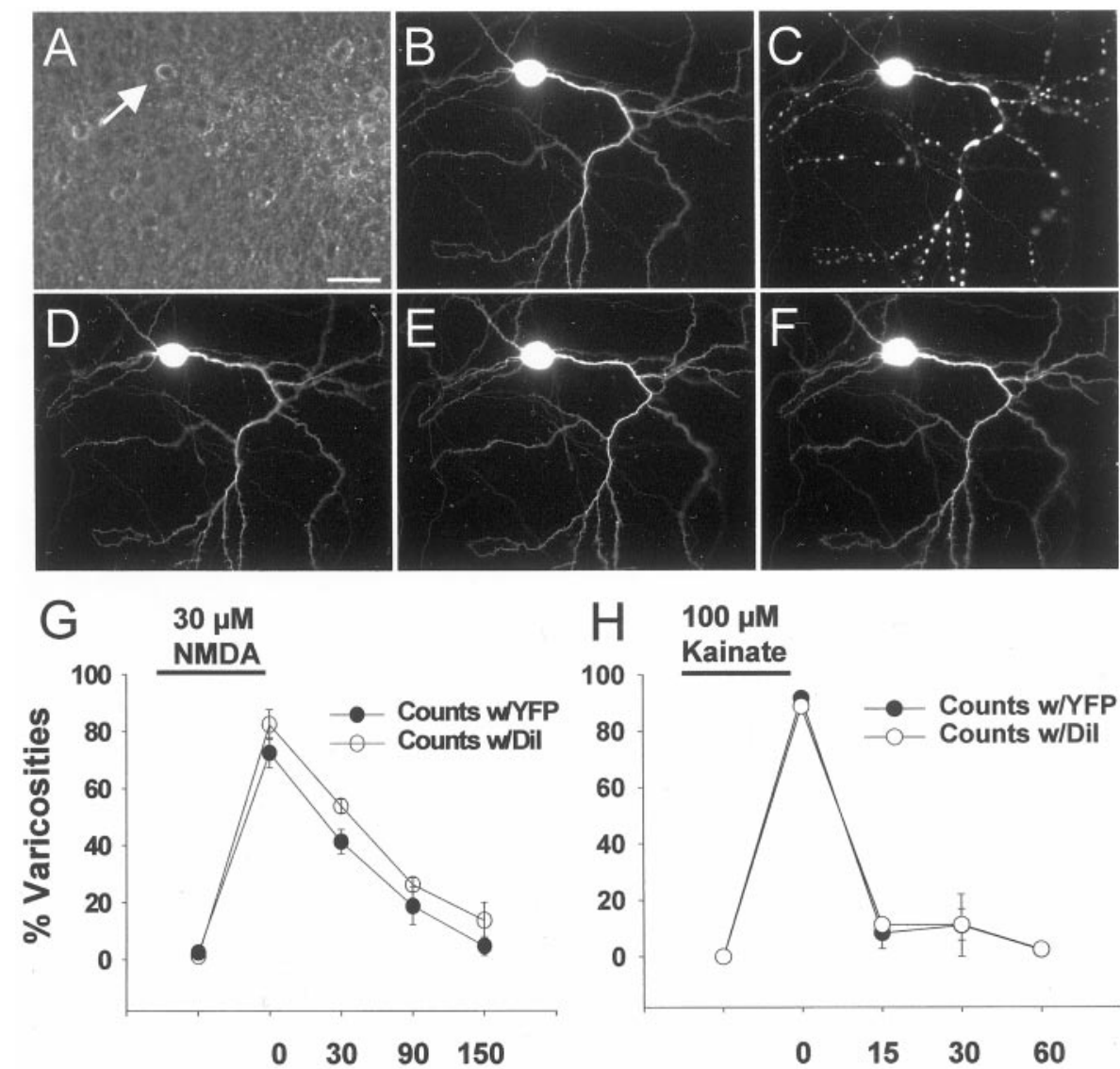

Recovery, min.
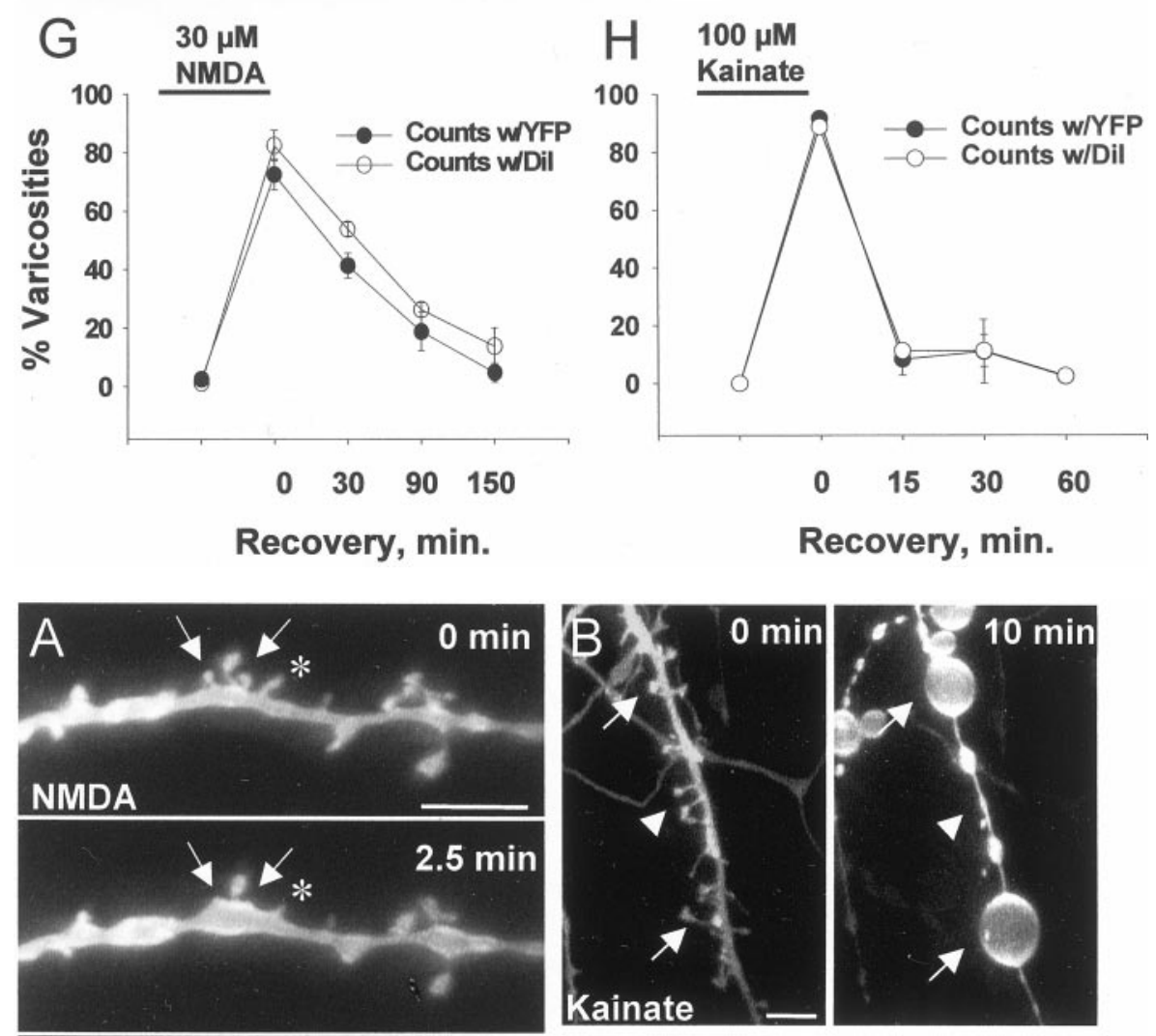

Recovery, min.
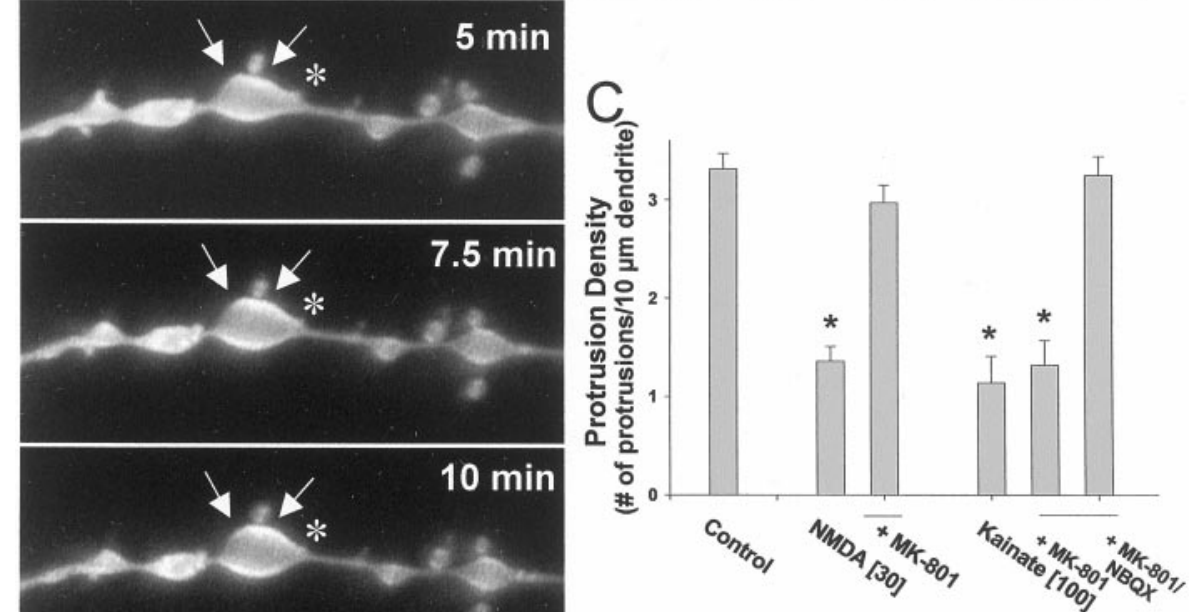

Exposure Condition (10 min)
Figure 2. Glutamate receptor activation triggers reversible dendritic swelling. $A-F$, Cortical neuronal cultures were transfected with YFP at day 2 and examined at day 15 in vitro. $A$, Phase-contrast image. Arrow identifies transfected neuron. $B$, Application of $30 \mu \mathrm{M}$ NMDA for 10 min produced localized dendritic varicosities $(C)$, which were no longer present at $1(D), 12(E)$, and $24 \mathrm{hr}(F)$ after agonist washout. $G, H$, YFP expression does not alter kinetics of dendritic injury and recovery. Cortical cultures transfected with YFP were exposed to $30 \mu \mathrm{M}$ NMDA $(G)$ or $100 \mu \mathrm{M}$ kainate $(H)$ for $10 \mathrm{~min}$ and fixed at indicated times after agonist removal. Fixed cultures were randomly labeled with DiI, and the percentage of neurons with dendritic varicosities was determined by YFP or DiI fluorescence. The $y$-axis is percentage of varicosities for both $G$ and $H$. Values represent mean \pm $\operatorname{SEM}(n=4$ cultures for each condition). Scale bar, $50 \mu \mathrm{m}$.

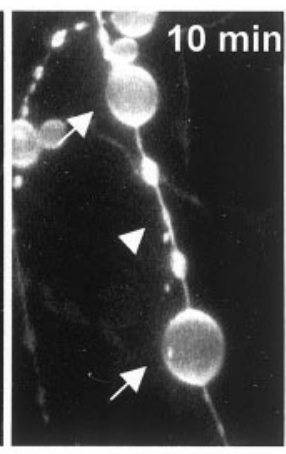

Figure 3. Time-lapse imaging reveals progression of spine loss during glutamate receptor activation. $A$, DiI-labeled neuron was imaged during exposure to $30 \mu \mathrm{M}$ NMDA. Symbols indicate representative spines that disappear. Asterisk shows a spine that retracted over $7.5 \mathrm{~min}$. $B$, Similar spine loss was observed in identified dendritic segment exposed to $100 \mu \mathrm{M}$ kainate. Spine loss occurred at sites of varicosity formation (arrows) and at intervening constricted segments (arrowheads). C, Protrusion densities (dendritic spines + filopodia) were measured in sister cultures exposed to wash conditions, $30 \mu \mathrm{M}$ NMDA for $10 \mathrm{~min}$ with or without $10 \mu \mathrm{M} \mathrm{MK}-801$, or to $100 \mu \mathrm{M}$ kainate for 10 min with or without $10 \mu \mathrm{M}$ MK-801 and $30 \mu \mathrm{M}$ NBQX. NMDA-induced loss of dendritic protrusions was blocked with MK-801 $(n=10$ cells, $p<$ $0.01)$. Kainate-induced protrusion loss was blocked by NBQX but not by MK-801 $(n=10$ cells, $p<0.01)$. Scale bar, $5 \mu \mathrm{m}$. 
Figure 4. Spine density recovers after sublethal excitotoxic or hypoxic exposure. $A$, Representative DiI-labeled dendrites are shown from sister cultures exposed to wash conditions, $30 \mu \mathrm{M}$ NMDA for $10 \mathrm{~min}$, or NMDA followed by $2 \mathrm{hr}$ recovery. $B$, Protrusion densities were measured in cultures exposed to wash, $30 \mu \mathrm{M}$ NMDA, or $100 \mu \mathrm{M}$ kainate for $10 \mathrm{~min}$ and permitted to recover for indicated periods before fixation and DiI labeling. MK-801 $(10 \mu \mathrm{M})$ was included in the kainate-treated groups to block possible NMDA receptor activation by endogenous glutamate release. Protrusion density was stable over $24 \mathrm{hr}$ in control conditions. Protrusion density decreased immediately after NMDA or kainate application and recovered to control levels within $30 \mathrm{~min}$ (kainate) or $2 \mathrm{hr}$ (NMDA). Values are mean \pm SEM $(n=30-55$ cells pooled from 6 independent experiments). $C$, Protrusion density was significantly decreased after oxygen glucose deprivation ( $25 \mathrm{~min}$ ) but recovered to control values within $24 \mathrm{hr}(n=20$ neurons pooled from two independent experiments). ${ }^{*} p<0.05$ compared with matched control condition. Scale bars, $5 \mu \mathrm{m}$.
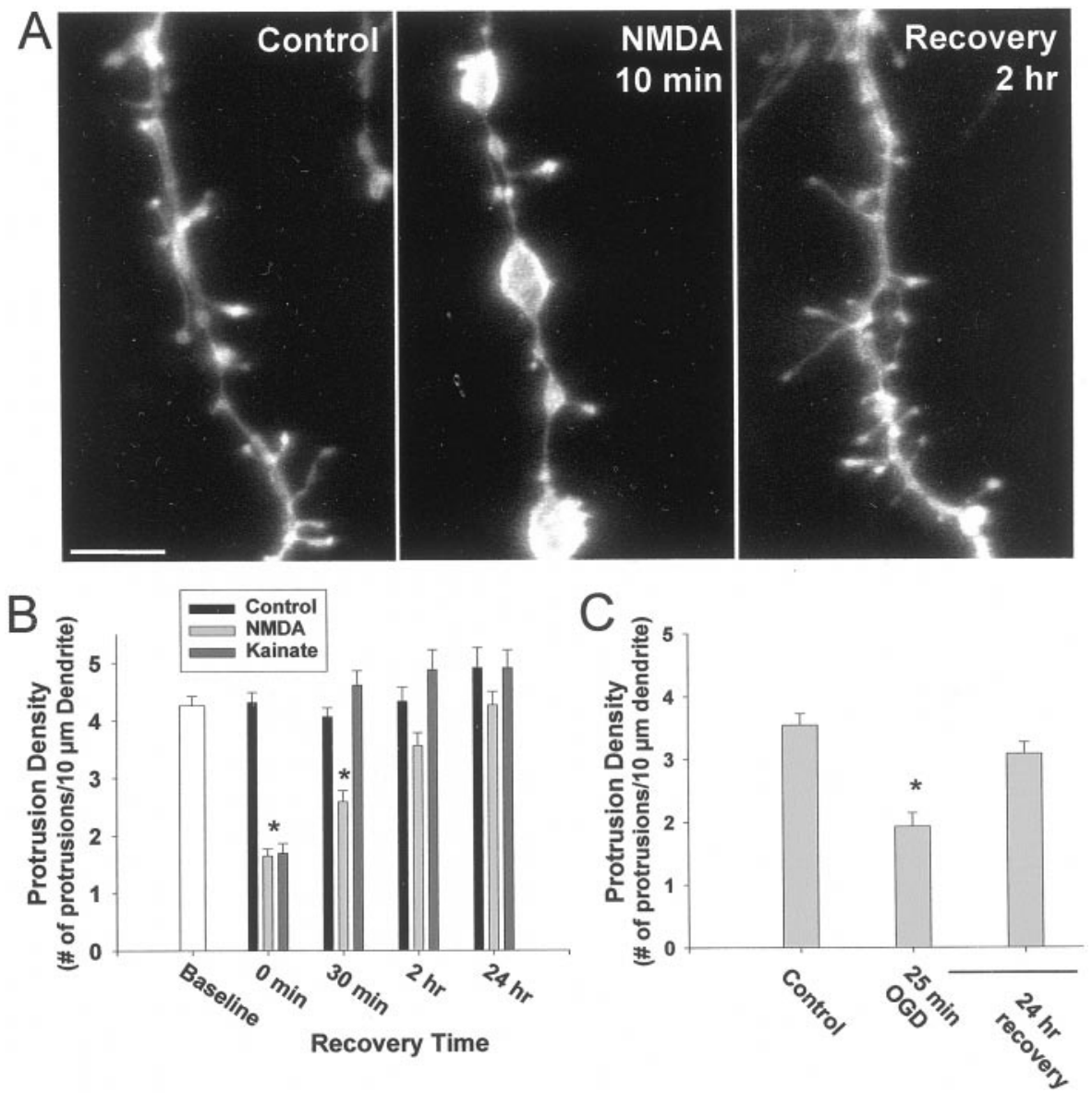

morphology would not influence the counts of spine-like structures. Dendritic segment length and protrusion counts were assessed on twodimensional maximal intensity projection images, and the presence or absence of individual protrusions was verified by simultaneous reference to the raw, unenhanced three-dimensional image planes. Therefore, the analysis included protrusions above or below the plane of the parent dendrite. Protrusion density was expressed as the number of protrusions per $10 \mu \mathrm{m}$ dendrite length. Each cell was counted as an individual observation $(n=1)$. Statistical differences in protrusion density were determined by one-way ANOVA followed by appropriate post hoc comparison (SigmaStat 2.0; Jandel Scientific, San Rafael, CA). Images were contrast enhanced for preparation of the final publication figures (Adobe Photoshop), using identical settings for each image in a given sequence.

\section{RESULTS}

\section{Characterization of primary cortical cultures and labeling methods}

Phase-contrast imaging of dissociated cortical cultures at day $15 \mathrm{in}$ vitro revealed densely spaced neuronal cell bodies in clusters $\left(5-10 \times 10^{5}\right.$ neurons/cm $\left.{ }^{2}\right)($ Dugan et al., 1995) but few details of neuronal morphology (Fig. $1 A$ ). Neuronal dendrites and axons were well visualized by application of DiI, as described previously (Park et al., 1996; Hasbani et al., 1998). Because DiI causes neuronal damage in long-term studies, we alternatively labeled neurons by transfection with the yellow-shifted green fluorescent protein variant, YFP. Liposome-mediated delivery of the YFP plasmid at $2 \mathrm{~d}$ in vitro resulted in bright, sustained YFP expression in a small subset of neurons $(<0.01 \%)$. YFP fluorescence (Fig. $1 B)$ and DiI labeling (see Figs. 3, 4) (Park et al., 1996) demonstrated intricately branched dendritic arbors studded with protrusions that included mature spines and few filopodia. Axons could be observed traveling great distances from their cell bodies.
Axonal varicosities, putative sites of neurotransmitter release, were observed at high magnification.

The average density of dendritic protrusions (spines + filopodia) was three to four per $10 \mu \mathrm{m}$ dendritic length (Fig. $1 C$ ). These values are similar to previous reports in primary hippocampal culture (Papa et al., 1995; Ziv and Smith, 1996). Approximately $10 \%$ of cortical neurons lacked protrusions (data not shown). For the present studies, population experiments (Figs. $1 C, 4,8$ ) included all neurons, but prospective time-lapse studies of labeled cells excluded neurons without spines (see Figs. 3, 6, 7, 9, 10). YFP transfection did not alter dendritic spine density (Fig. 1C) or intracellular calcium elevation during application of NMDA (Fig. 1D).

\section{Dendritic swelling and recovery after sublethal glutamate receptor activation}

Application of the glutamate receptor agonists NMDA $(30 \mu \mathrm{M})$ or kainate (100-300 $\mu \mathrm{M}$ ) for $10 \mathrm{~min}$ (Fig. 2) or exposure to $25 \mathrm{~min}$ of combined oxygen-glucose deprivation (data not shown) (Park et al., 1996) induced focal swellings along the length of the neuronal dendrites in $>90 \%$ of the cells (Fig. 2C,G,H). These exposure conditions were associated with $<20 \%$ cellular death by the following day (assessed by propidium iodide exclusion) (Hasbani et al., 1998). Although dendritic varicosities appeared discontinuous in YFP-expressing neurons, labeling with DiI showed that swollen dendritic segments remained connected by thin strands of intervening membrane (Fig. 3A,B) (Park et al., 1996). Varicosities resolved spontaneously over $30 \mathrm{~min}$ to $2 \mathrm{hr}$ after agonist removal, with a slower time course after NMDA receptor activation than after AMPA/kainate receptor activation (Fig. 


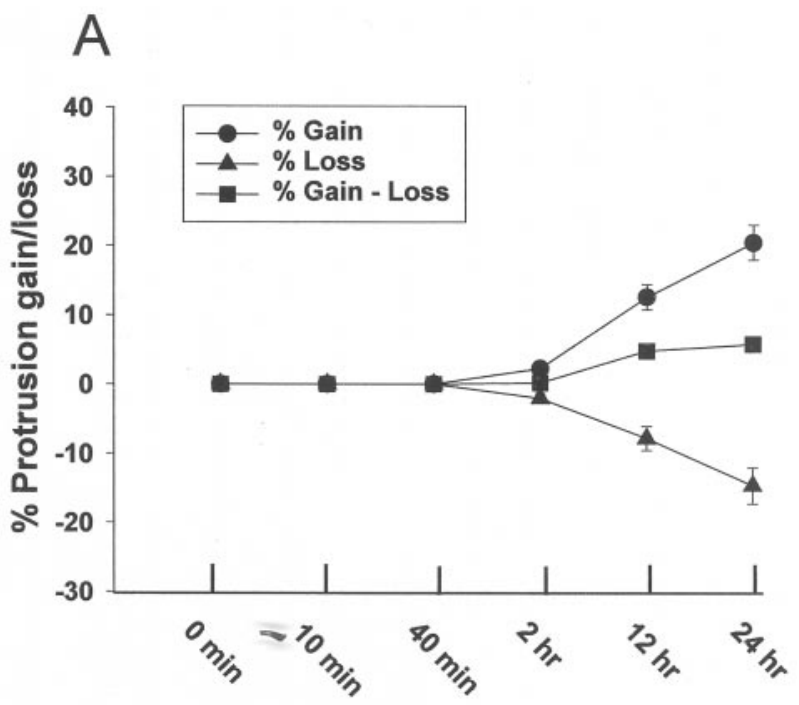

B

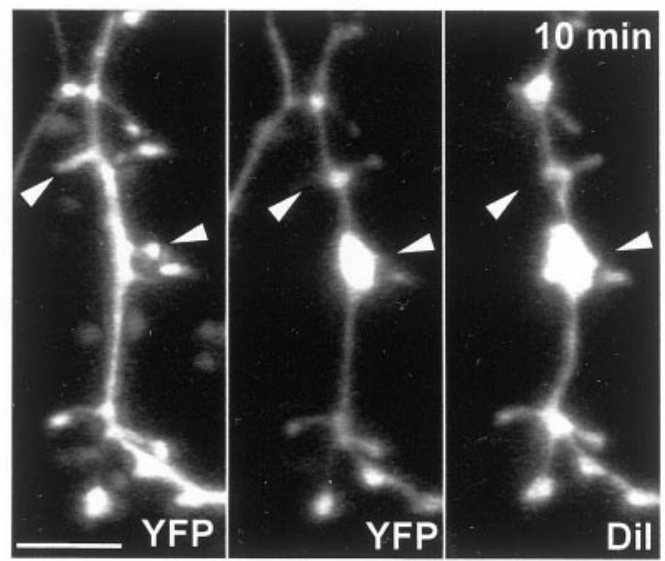

Figure 5. Protrusion turnover and NMDA-induced spine loss visualized with YFP. $A$, Basal turnover of dendritic protrusions was low in $15 \mathrm{~d}$ cortical neuronal cultures. Confocal images of YFP-expressing dendrites were obtained at initial time point and were repeated after $10 \mathrm{~min}$ to 24 hr under control conditions. The percentage of protrusion gain or loss was calculated by dividing the cumulative number of protrusions that appeared or disappeared at each time point by the number of protrusions at the initial time point. Note minimal gain or loss during initial $2 \mathrm{hr}$ of observation. Values are mean $\pm \operatorname{SEM}(n=21$ secondary dendrites of 10 neurons from three different culture platings). $B$, DiI labeling confirms spine loss imaged with YFP. A YFP-labeled dendritic segment is shown before and after exposure to $30 \mu \mathrm{M}$ NMDA for $10 \mathrm{~min}$. The culture was fixed immediately, and the cell was labeled with DiI by micropipette. Arrowheads note positions of lost spines. Scale bar, $5 \mu \mathrm{m}$.

2D, $G, H$ ) (Faddis et al., 1997; Hasbani et al., 1998). The time course and intensity of dendritic swelling and recovery were not altered by YFP transfection (assessed in sister cultures labeled with DiI after fixation). Because little cellular death was observed under these conditions, YFP-transfected neurons could be studied to determine the lasting fate of injured dendrites (Fig. 2E,F).

\section{Spine loss and recovery after excitotoxic exposure}

We used both time-lapse and population studies to examine spine loss during glutamate agonist application. DiI-labeled neurons were exposed to NMDA or kainate and imaged every 2.5 min for 10 min (Fig. 3). As shown in Figure 3A, many dendritic spines were lost as dendritic swelling developed, sometimes disappear- ing over several minutes of agonist exposure (Fig. $3 A$, asterisk). It often appeared that spines were enveloped by focally swollen dendrites. Indeed, most spines were observed to be lost as sites of local dendritic swelling; however, spine loss also occurred distant from varicosities (Fig. 3B, arrowhead). Protrusion density was reduced to a similar extent with exposure to NMDA or kainate and was preserved by coapplication of receptor antagonists MK801 or NBQX, respectively (Fig. 3C).

To assess the fate of dendritic protrusions after agonist removal, we measured protrusion density in sister cultures that were exposed to NMDA, kainate, or oxygen-glucose deprivation, and labeled with DiI after fixation immediately after exposure or at various time points over the subsequent $24 \mathrm{hr}$ (Fig. 4). Protrusion density returned to baseline within $30 \mathrm{~min}$ to $2 \mathrm{hr}$ after NMDA or kainate removal and was stable over the next $24 \mathrm{hr}$. Recovery was slower after NMDA receptor activation than after AMPA/kainate receptor activation (Fig. 4B). Neurons exposed to oxygenglucose deprivation for $25 \mathrm{~min}$ also underwent a reversible $50 \%$ reduction in protrusion density (Fig. $4 C$ ).

\section{Observation of identified dendritic spines}

These population studies demonstrate near-complete recovery of protrusion numbers but do not address the location of recovered protrusions or their morphology. To answer these questions, we used time-lapse microscopy of YFP-labeled neurons.

We first demonstrated that YFP is a viable marker for observing spines over time and for demonstrating spine loss. We examined the stability of identified spines under basal conditions by capturing consecutive time-lapse pictures of identified dendritic segments labeled with YFP (Figs. 5A, 6A, Control). In agreement with published studies, the rate of protrusion turnover was low (Okabe et al., 1999). In normal culture medium, the overall density of dendritic protrusions remained relatively constant, increasing slightly over $24 \mathrm{hr}$ (Fig. 4B, Control); this net stability reflected a $20.5 \pm 2.5 \%$ gain of new protrusions and $14.7 \pm 2.6 \%$ loss of existing protrusions (Fig. $5 A$ ). Protrusions were especially stable over the first $2 \mathrm{hr}$ of imaging with little gain or loss $(<2.5 \%$ gain or loss) (Figs. 5A, 6A, Control).

Changes in cytosolic YFP distribution or fluorescence intensity might produce an artifactual appearance of spine loss. To verify that disappearance of spine fluorescence after NMDA exposure reflected spine loss rather than YFP redistribution or fading, YFP-labeled dendrites were fixed and relabeled with the membrane tracer, DiI (Fig. 5B). In a series of 13 consecutively doublelabeled neurons, 54 of $63(86 \%)$ YFP-labeled spines that disappeared during NMDA or kainate exposure were also absent by DiI labeling. Use of YFP fluorescence may result in, at most, a small population of spines that are misclassified as absent. These data indicate that agonist-induced spine loss can be demonstrated using YFP as well as DiI.

We assessed the fate of identified spines in YFP-labeled neurons by time-lapse confocal microscopy. As mentioned above, protrusion locations remained stable over several hours of imaging under control conditions (Fig. 6A), although individual spine morphology was highly motile (data not shown) as observed by Fischer et al. (1998). In the minutes to hours after NMDA or kainate exposure, spines that had disappeared were observed to reappear in their previous locations. Spine recovery occurred by outgrowth from regions of dendritic swelling and from the intervening dendritic segments (Fig. 6A, NMDA, asterisks). Recovering spines initially lacked heads in many cases but rapidly developed a mature morphology (length $<4 \mu \mathrm{m}$, presence of heads). 
Control

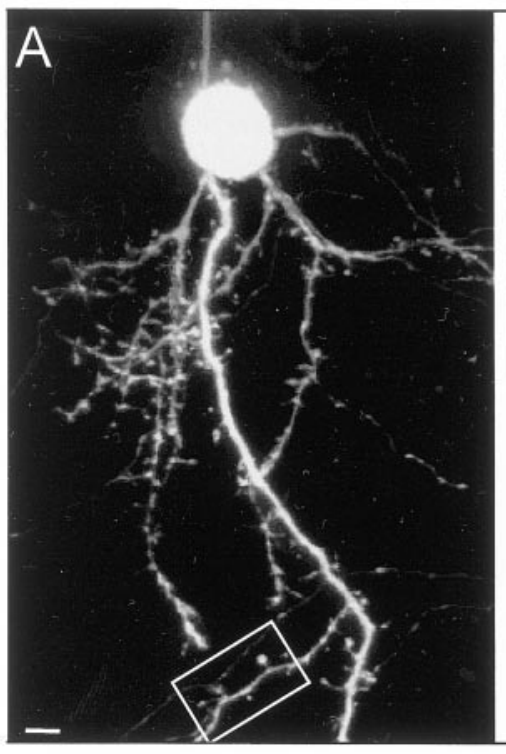

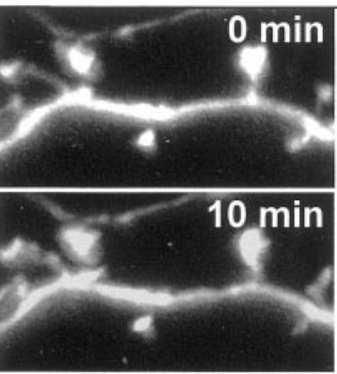
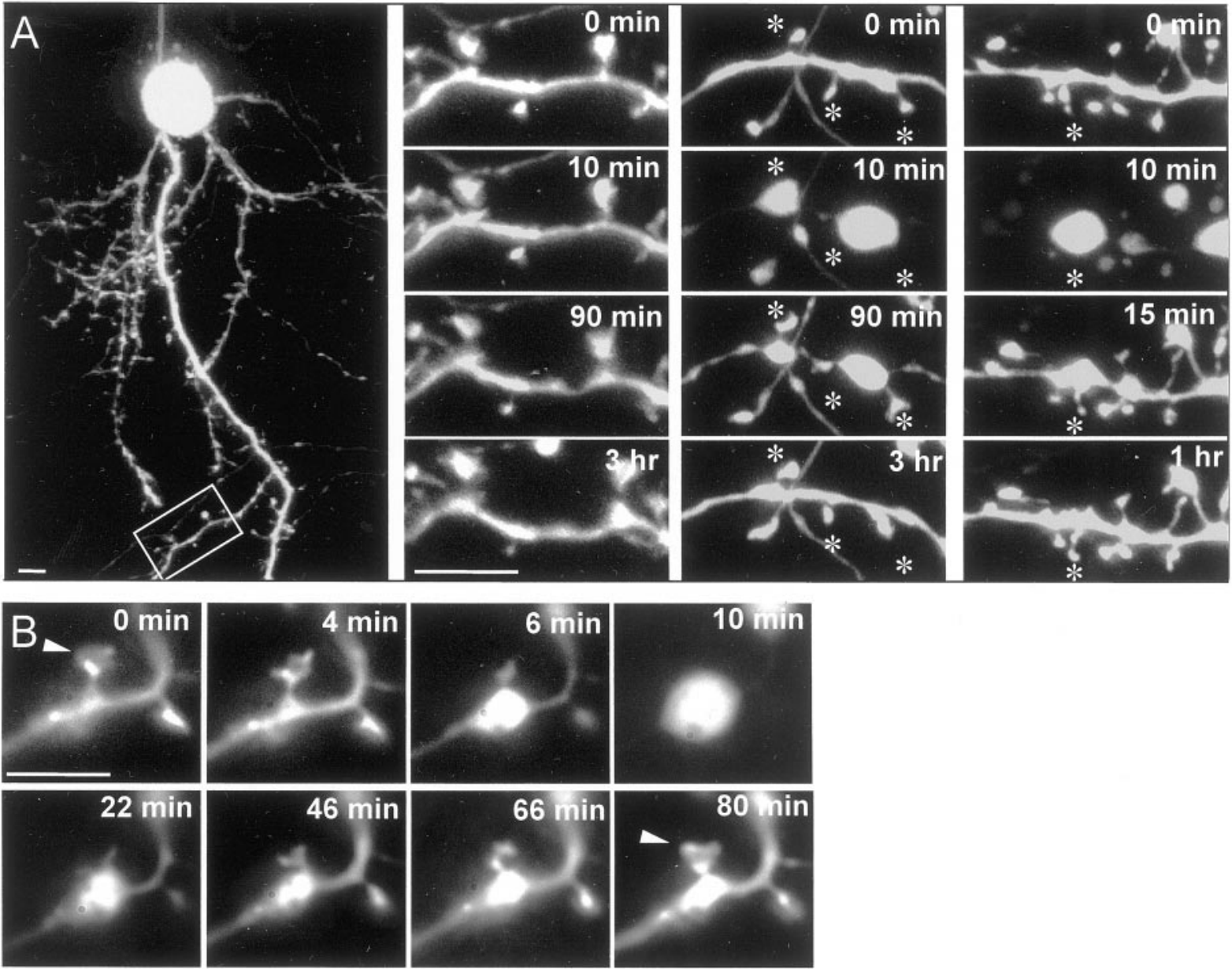

Figure 6. Dendritic spines recover rapidly after excitotoxic exposure. $A$, Time-lapse series of YFP-transfected neuron under control conditions shows spine stability over $3 \mathrm{hr}$ (box shows region for control series). Treatment with either $30 \mu \mathrm{M}$ NMDA or $100 \mu \mathrm{M}$ kainate resulted in rapid loss of dendritic spines (asterisks). Spines recovered over the subsequent 1-3 $\mathrm{hr}$ in the same locations from which they disappeared. $B$, Imaging of YFP-labeled dendrite during and after treatment with $100 \mu \mathrm{m}$ kainate for $10 \mathrm{~min}$. Sequential images show dynamic process of loss and recovery of an identified dendritic spine (arrowheads). Scale bars, $5 \mu \mathrm{m}$.

Spines were highly motile during recovery, and spine heads often exhibited small dynamic extensions before a final stable morphology was achieved (Fig. 6B). The majority of spines reemerged within $1 \mu \mathrm{m}$ of their original locations $(n=41$ of 43 spines after NMDA and 87 of 88 spines after kainate). In agreement with our population studies (Fig. $4 B$ ), time-lapse images showed that spine recovery occurred between 1 and $3 \mathrm{hr}$ after NMDA treatment and between 30 and 60 min after kainate treatment (Fig. 6A).

Although the majority of spines reemerged in locations from which they disappeared, we also observed the new appearance of a subset of longer protrusions after glutamate receptor activation. Spines were defined as structures with thin necks and well defined heads, and filopodia were classified as thin structures $>4 \mu \mathrm{m}$ in length and lacking heads (Ziv and Smith, 1996) (see Materials and Methods). At basal conditions on days $15-16$ in vitro, $\sim 85 \%$ of dendritic protrusions were classified as spines and $\sim 15 \%$ of protrusions were classified as filopodia (Figs. $4 B, 7 A$; values derived from the same data set). Filopodia $<4 \mu \mathrm{m}$ were rare ( $<1 \%$ of all protrusions; data not shown). A net increase in filopodia density was observed in cultures recovering from excitotoxic injury (Fig. 7A). However, $24 \mathrm{hr}$ after injury, the density of filopodia (Fig. 7A) and spines (data not shown) was no longer significantly different from baseline conditions. Time-lapse microscopy revealed the appearance of filopodia at sites where spines were previously located and, occasionally, in new locations (Fig. 7B). Filopodia formation did not represent a primary mechanism by which most spines recovered after excitotoxic injury; most spines acquired a mature morphology without forming longer intermediate structures over the 30-120 min period of observation.

\section{Actin depolymerization does not prevent spine recovery}

Actin is the major cytoskeletal element of dendritic spines (Fifkova and Delay, 1982) and is thought to be important for determining spine shape and motility (Fischer et al., 1998). We hypothesized that spine recovery after excitotoxic injury would be prevented under conditions of actin depolymerization. We examined this hypothesis by pretreating cultures with latrunculin-A, a toxin that inhibits actin assembly by sequestering monomeric actin (G-actin), resulting in net depolymerization of actin polymer (F-actin). Application of $1 \mu \mathrm{M}$ latrunculin-A for $2 \mathrm{hr}$ was 


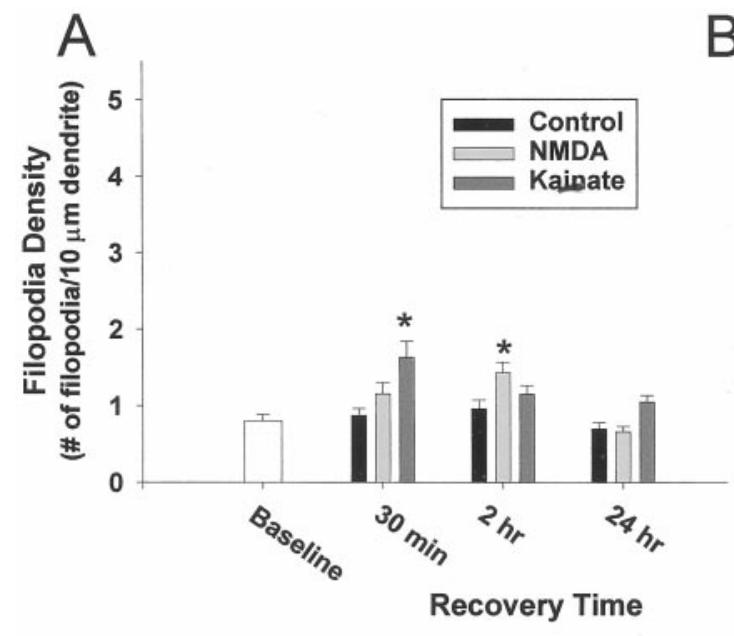

sufficient to eliminate filamentous actin in dendrites and spines, as assessed by staining with fluorescent phalloidin (Fig. 8B). In agreement with Fischer et al. (1998), we found that inhibition of actin polymerization blocked spine motility (observed in timelapse images at $20 \mathrm{sec}$ intervals; data not shown) but did not cause spine loss (Fig. 8B). Pretreatment and co-treatment with latrunculin-A did not prevent spine loss or recovery after $30 \mu \mathrm{M}$ NMDA or $300 \mu \mathrm{M}$ kainate for $10 \mathrm{~min}$ (Fig. 8C,D). Experiments performed with a $12 \mathrm{hr}$ pretreatment of latrunculin-A or with as much as $10 \mu \mathrm{M}$ latrunculin-A also demonstrated fully reversible excitotoxic spine loss (data not shown). These results suggest that although polymerization of the actin cytoskeleton contributes to spine motility, it is not required for either spine loss or recovery.

\section{Spines remain tethered to presynaptic terminals during spine loss and recovery}

One can envision several outcomes when spines disappear: spines may become physically separated from presynaptic boutons, boutons may be lost altogether, or spines and boutons may remain adjoined. As spines recover after injury, they may associate again with presynaptic terminals or may be left devoid of synaptic contacts. These possibilities have different consequences for synaptic function during and after injury. To distinguish between the possibilities, we used DiI to label a random subpopulation of neurons in cultures expressing YFP. This procedure allowed us to identify points of synaptic contacts between two cells, one labeled with YFP and the other with DiI (Fig. $9 A-C$, arrows and arrowheads). Dendrites and axons were imaged and then exposed to either $30 \mu \mathrm{M}$ NMDA or $100 \mu \mathrm{M}$ kainate and re-imaged after 10 min. Presynaptic terminals remained in close proximity to the postsynaptic membrane during spine loss induced by either NMDA or kainate (Fig. 9D-F, arrow).
As a second measure of presynaptic location during spine loss, we performed immunocytochemistry against the synaptic vesicle protein, synapsin. Under control conditions, most spine heads were associated with synapsin staining (Fig. 9G) $(91 \% ; n=$ $333 / 365$ spines) and only a small percentage of spines $(7 \% ; n=$ $24 / 365$ spines) had associations at their base (along the dendrite shaft, within $1 \mu \mathrm{m}$ of the spine neck). After excitotoxic injury, synapsin-positive puncta were observed along the dendrite shafts within $1 \mu \mathrm{m}$ of the location of the lost spine (Fig. 9H,I) $(n=$ 16/17 spines after NMDA and 17/18 spines after kainate). These observations show that when spines disappear, their synaptic contacts are not lost but rather persist near the dendritic shaft. Furthermore, most spines that underwent loss were associated with synapsin staining on spine heads after recovery (Fig. 10) ( $n=39 / 41$ spines after NMDA and 22/23 spines after kainate). Immunolabeling against a second synaptic vesicle protein, synaptophysin, yielded the same results (data not shown), further suggesting that recovered spines are synaptically connected.

\section{DISCUSSION}

Acute dendritic swelling and spine loss are pathological hallmarks of excessive glutamate receptor activation, or excitotoxicity, and occur after ischemia, trauma, or epilepsy (Olney, 1971; Kolb and Gibb, 1993; Rowntree and Kolb, 1997; Jiang et al., 1998). In the current experiments, sublethal glutamate receptor activation resulted in spine loss associated with focal dendritic swelling. Spines recovered spontaneously within $2 \mathrm{hr}$ after agonist removal. Synaptic connections were preserved despite an overwhelming change in dendrite morphology. Although spine loss in experimental models is often considered to reflect long-term synaptic 
damage, this process may be rapidly reversible under certain conditions.

\section{Rapid recovery of dendritic spines after glutamate receptor activation}

Recovery of spine density has been observed over days to weeks in injury models (Kolb and Gibb, 1993; Muller et al., 1993; Akulinin et al., 1997). We were surprised to observe that spine loss and recovery could occur over much shorter intervals. Rapid alterations in dendritic spine morphology or numbers have been described in other settings such as LTP (Engert and Bonhoeffer, 1999) and synaptic activation (Toni et al., 1999) or after slice preparation from postnatal or adult rat brain (Kirov et al., 1999). However, this is the first description of rapid spine reformation, a process that was complete within 15-120 min after spine loss. Spine turnover triggered by glutamate receptor activation may occur under physiological conditions of intense synaptic activity or in acute excitotoxic brain injury.

We used complementary methods to demonstrate spine loss and recovery. Protrusion density was assessed in neurons fixed after sublethal treatment and post-labeled with DiI, and individual spines were observed by high-resolution time-lapse microscopy in neurons expressing YFP or labeled with DiI. These overlapping methods excluded potential artifacts from phototoxicity, dye toxicity, or fluorophore redistribution, and demonstrated widespread excitotoxic spine loss in agreement with previous in vitro and in vivo results. Baseline spine density and the time course of spine loss and recovery were quantitatively similar with all techniques. Time-lapse microscopy of labeled neurons allowed important additional observations. First, protrusion density was stable, and turnover did not occur under control conditions during a $2 \mathrm{hr}$ observation period (Figs. 5A, 6A, Control). These results confirm that dendritic spines in cultured neurons are stable after 12-14 d in vitro (Papa et al., 1995; Ziv and Smith, 1996; Okabe et al., 1999). Although spine development was largely complete at the time of study in these cultures, it is possible that a propensity for rapid recovery may be unique to maturing neurons. Second, time-lapse studies clearly demonstrated loss of spines in identified dendritic segments (Figs. 3, 5B, 6 ), and allowed experiments matching lost spines with synaptic terminals (Figs. 9, 10). Finally, these studies allowed direct visualization of the location of emerging spines in relation to their disappearance (Fig. 6).

Spines might emerge in new or previous locations, and these possibilities have distinct implications for synaptic connectivity. We occasionally observed elaboration of spine filopodia in new locations on recovering dendrites after either NMDA or kainate application (Fig. 7). Emergence of dendritic filopodia has been observed to initiate synapse formation in the developing nervous system (Ziv and Smith, 1996) and has been observed after highfrequency stimulation in developing hippocampal slice cultures (Maletic-Savatic et al., 1999). Emergence of new filopodia was not a frequent observation and did not constitute the primary mechanism by which spine density recovered in this model; rather most spines recovered at their original sites (Fig. 6).

The observed alterations in dendritic morphology raise the possibility that presynaptic and postsynaptic elements might become structurally separated. However, double-label time-lapse studies showed that synaptically paired neurons remained in contact despite dendritic swelling and spine loss (Fig. 9A-F), and synapsin-immunoreactive puncta were observed in proximity to postsynaptic dendrites even during acute spine loss (Fig. 9H,I).
These results parallel electron microscopic observations of Olney and colleagues in excitotoxic or ischemic brain lesions in vivo (Olney, 1971; Olney et al., 1979; Ikonomidou et al., 1989), which demonstrated intact presynaptic terminals in opposition to markedly swollen postsynaptic dendrites. Thus, spine loss need not
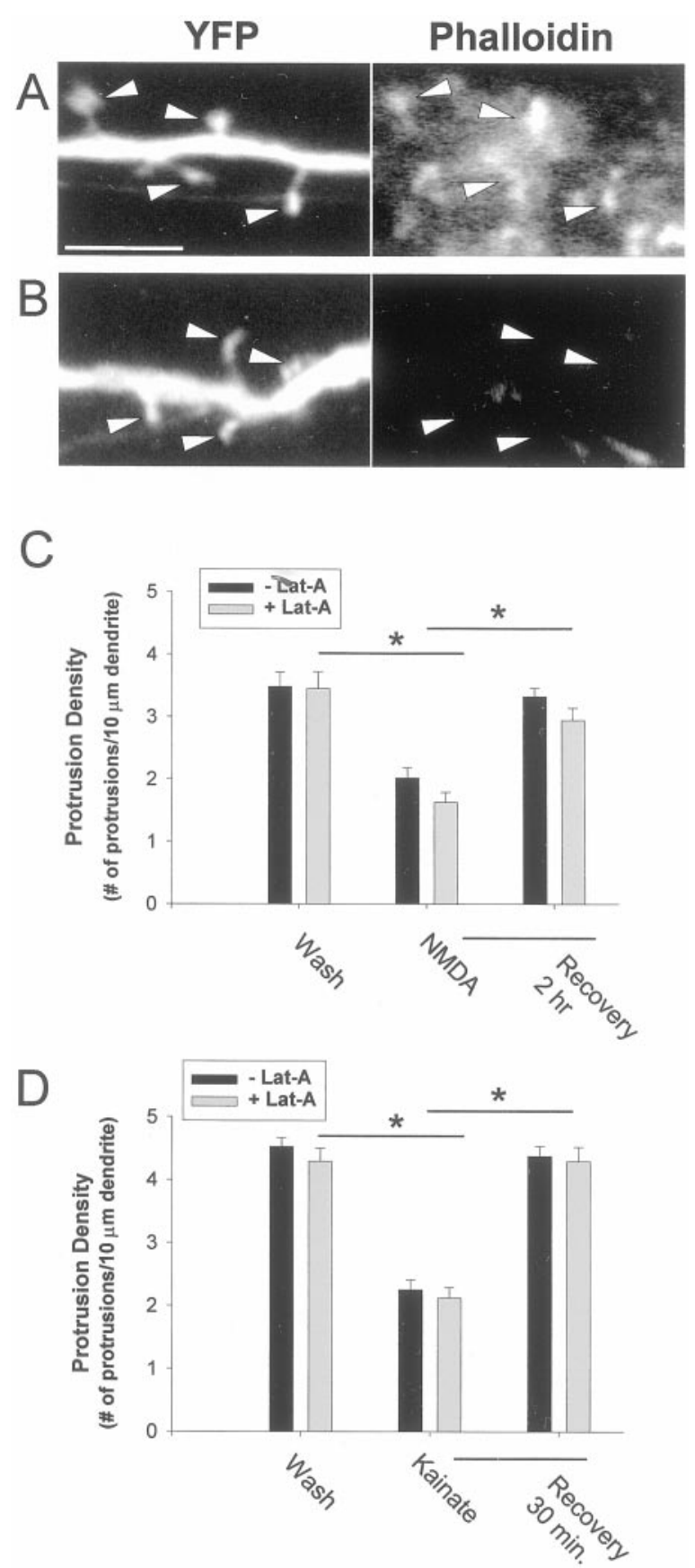

Figure 8. Spine recovery is not blocked by actin depolymerization. $A, B$, Neurons expressing YFP were exposed to latrunculin-A (1 $\mu \mathrm{M}$ for $2 \mathrm{hr})$ or vehicle. Filamentous actin visualized by phalloidin conjugated to Alexa568 colocalized with spine heads under control conditions ( $A$, arrowheads) but was no longer detectable after latrunculin-A treatment $(B) . C, D$, Protrusion density was determined in cultures exposed to $30 \mu \mathrm{M}$ NMDA, $300 \mu \mathrm{M}$ kainate, or sham wash in the presence or absence of $1 \mu \mathrm{M}$ latrunculin-A (pretreated for $2 \mathrm{hr}$ ). Latrunculin-A did not significantly alter spine density, loss, or recovery. ${ }^{*} p<0.05(n=15-30$ cells pooled from 2-3 separate experiments). Scale bar, $5 \mu \mathrm{m}$. 


\section{Dendrite}

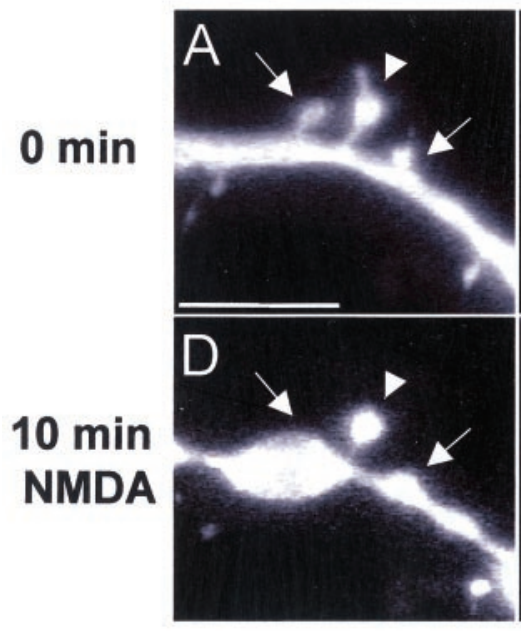

Axon
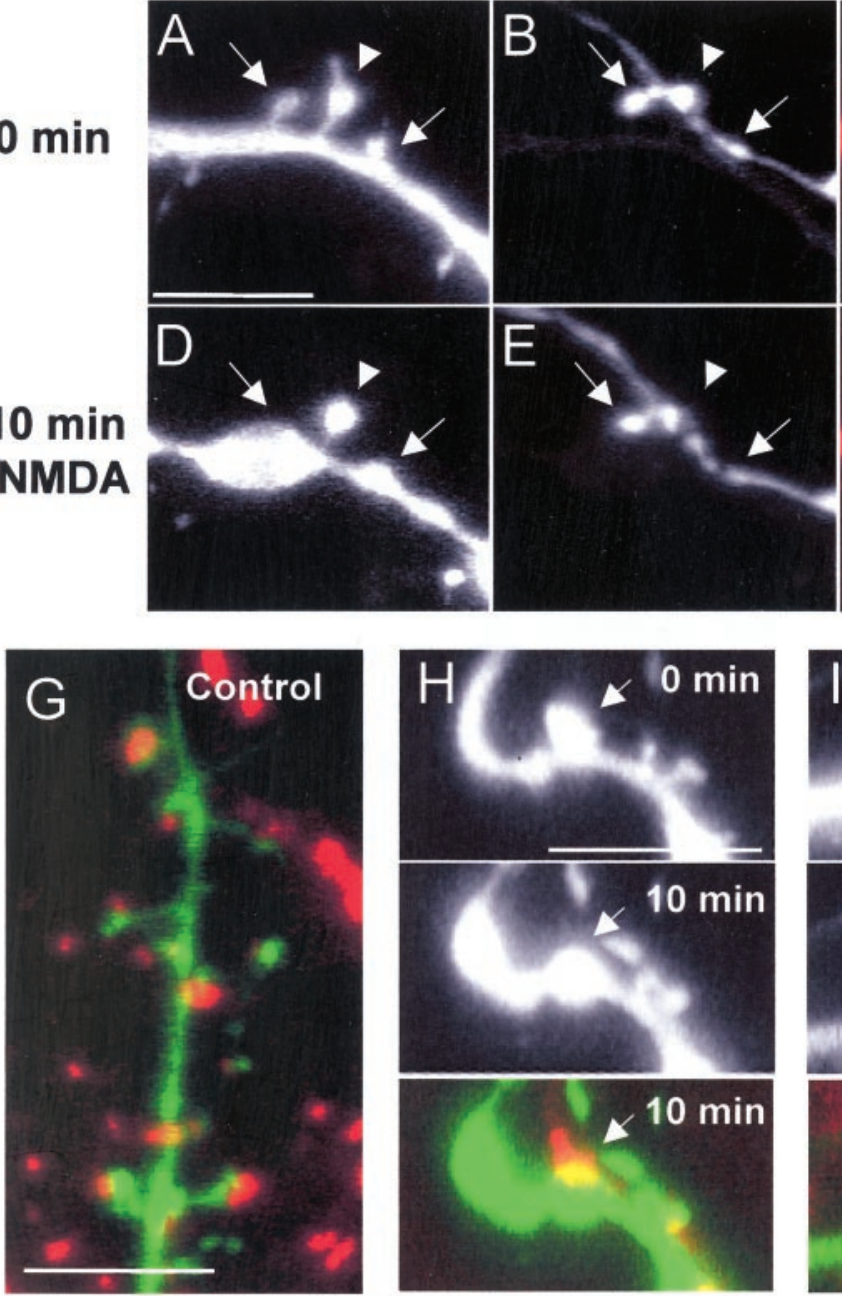

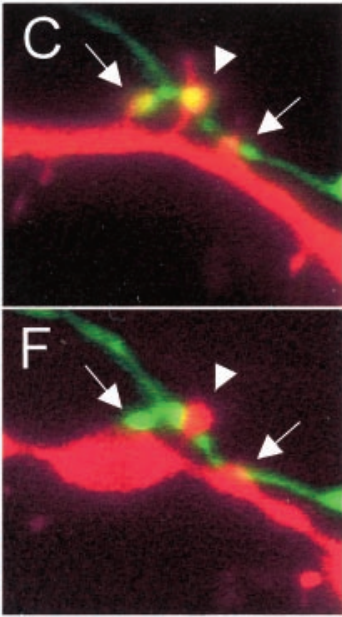

Merged

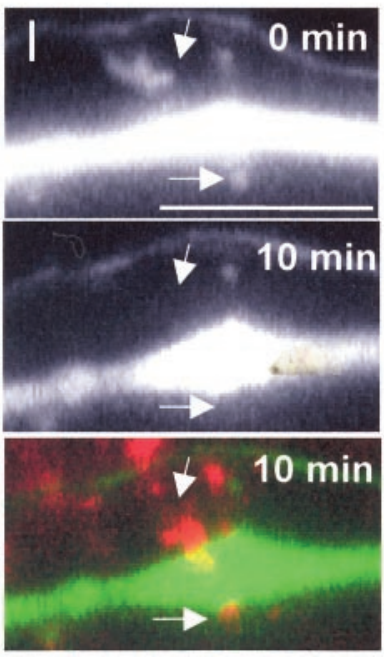

Figure 9. Presynaptic elements remain adjacent to the postsynaptic membrane during spine loss. Confocal images show paired neurons in which the postsynaptic dendrite is labeled with $\operatorname{DiI}(A)$ and presynaptic axon is labeled with YFP $(B)$. Merged images $(C)$ reveal sites of presumed synaptic contact (arrows, arrowhead). Spines were identified in three-dimensional stacks according to morphological criteria (see Materials and Methods). Exposure to NMDA for $10 \mathrm{~min}$ resulted in varicosity formation and spine loss $(D$, arrows $)$, but presynaptic boutons persisted next to the postsynaptic dendrite membrane at locations where spine loss occurred ( $E, F$, arrows). Results are representative of three similar experiments, each with NMDA or kainate. $G-I$, Synapsin-immunoreactive puncta are found on dendritic shafts at locations of spine loss. YFPlabeled neurons were fixed after wash treatment $(G)$ or visualized before and after addition of $30 \mu \mathrm{M}$ NMDA $(H)$ or $100 \mu \mathrm{M}$ kainate (I) for 10 min (top two images in $H$ and $I$ ). Immediately after agonist exposure, neurons were fixed and double-labeled with antibodies to YFP (green) and synapsin (red) (bottom images in $H$ and $I$ ). Synapsin-immunoreactive boutons were found on dendritic spine heads under wash conditions $(H)$ and on shafts at locations of spine loss (H, I, arrows). Scale bars, $5 \mu \mathrm{m}$. reflect loss of synaptic contacts. This may be an important consideration in studies of spine density in vivo.

\section{Mechanisms of spine recovery}

Most dendritic spines were lost at sites of varicosity formation. Moreover, spine loss and recovery had kinetics similar to that of varicosity formation and recovery (Figs. 2G,H, 4B), suggesting that spine loss may be the result of engulfment by swollen dendritic membrane. Therefore, it is possible that recovery may depend on cellular processes that drive restoration of dendritic shape after excitotoxic varicosity formation, such as volume regulatory pathways, calcium homeostasis, and cytoskeletal rearrangement (Faddis et al., 1997; Hasbani et al., 1998; Korkotian and Segal, 1999a,b; Segal et al., 2000).

Another intriguing possibility is that spine recovery involves factors independent of varicosity resolution. Time-lapse images demonstrated that spines recover through a dynamic process, whereby spines first protrude and then reestablish their morphology (Fig. 6B). We hypothesized that actin polymerization would be required for spine recovery. Actin is the major cytoskeletal element of dendritic spines (Fifkova and Delay, 1982) and is critical for spine motility (Crick, 1982; Fischer et al., 1998; Matus, 1999) and receptor localization (Allison et al., 1998; Sattler et al., 2000). However, pharmacological disruption of actin did not alter basal protrusion density (Kim and Lisman, 1999) or prevent recovery after glutamate receptor activation. Although NMDA receptor activity can be reduced by actin depolymerization (Rosenmund and Westbrook, 1993), latrunculin application did not decrease spine loss after NMDA application in our experiments. Latrunculin-A fully disrupted actin polymerization under our experimental conditions as evidenced by loss of spine motility and phalloidin staining. Taken together, these results suggest that actin is not required for reemergence of spines after glutamate receptor activation.

Our observations in cultured cortical neurons (Park et al., 1996) agree with reports of selective spine loss in hippocampal cultures after NMDA application (Halpain et al., 1998). However, the results differ in several respects. Compared with cortical cultures, hippocampal cultures (Allison et al., 1998; Halpain et al., 1998) appear to have more actin in dendritic spines, greater resistance of spine actin to latrunculin-A, and an absence of varicosity formation during NMDA exposure and spine loss. These differences, which we confirmed in low-density hippocampal cultures (Allison et al., 1998) prepared by A. M. Craig (our unpublished data), may be attributable to differences in tissue source, culture preparation, presence of astrocytes, or neuronal density. Spine loss associated with dendritic varicosity formation is well described in brain slice and in vivo models of excitotoxic and hypoxic-ischemic insults, and therefore dissociated cortical neu- 

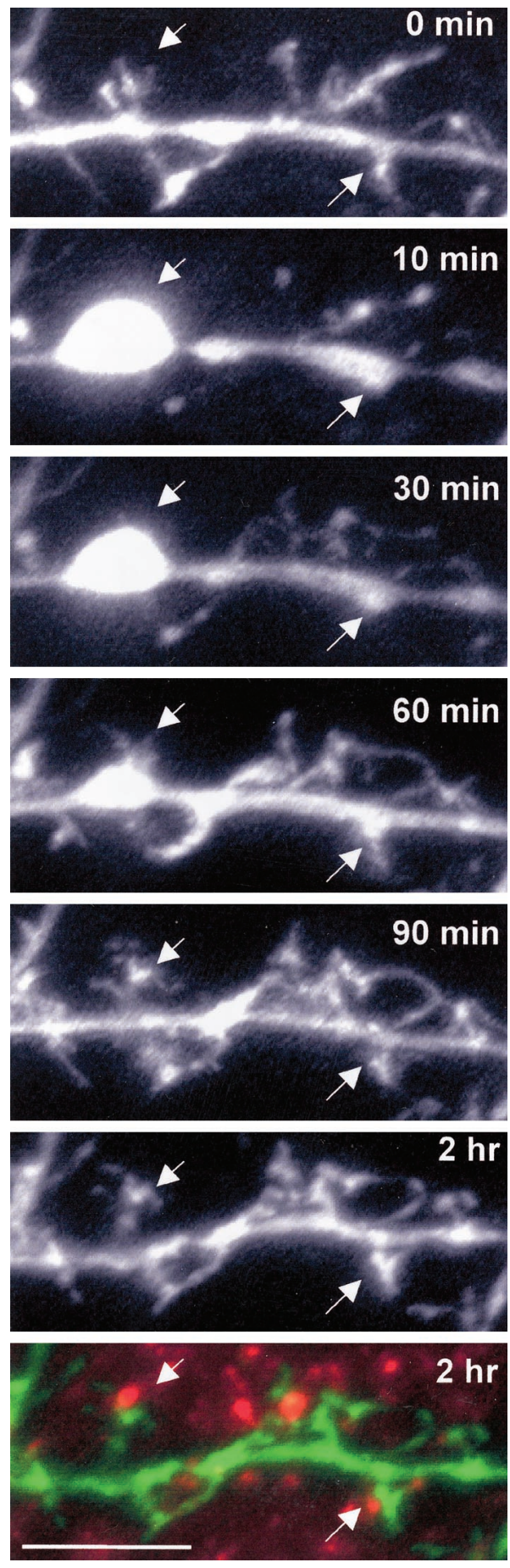

Figure 10. Synapsin-immunoreactive puncta are found on recovered spines. A YFP-labeled neuron was imaged at the time points indicated, during and after treatment with $30 \mu \mathrm{M}$ NMDA (added at time 0 min and washed out at time $10 \mathrm{~min}$ ). After $2 \mathrm{hr}$ of recovery, the culture was fixed and double-labeled with antibodies to YFP (green) and synapsin (red). Bottom image shows that synapsin-immunoreactive boutons were found on the heads of dendritic spines that underwent loss and recovery (arrows). Scale bar, $5 \mu \mathrm{m}$.

ronal cultures represent an appropriate model system for the present studies.

Dendritic spines most often reappeared in their original locations. How is this location information retained? Preservation of synaptic contacts during spine loss might help guide the location of spine recovery. Alternatively, important cytoskeletal components or structural proteins of the spine might be preserved, even when the spine membrane and spine cytosol are engulfed by the parent dendrite. For example, the postsynaptic density protein, PSD-95, and the NMDA receptor subunit, NR1, are not lost from postsynaptic spines in neurons exposed to latrunculin-A or NMDA, respectively, despite disruption of spine actin (Allison et al., 1998; Sattler et al., 2000). Preliminary observations show that actin and the actin-associated protein, drebrin, remain intact after NMDA-induced spine loss (our unpublished data). Residual core spine proteins could impart a structural or functional presence to guide spine reassembly after excitotoxic loss.

\section{Significance of transient spine loss and recovery}

Loss of dendritic spines has important consequences for neuronal function. Spine loss correlates with behavioral impairment after ischemia (Kolb and Gibb, 1993; Akulinin et al., 1997), perhaps by interfering with synaptic transmission or altering synaptic connectivity. Additional structural changes in dendrite shape, including formation of focal varicosities and constrictions, may lead to early dendritic transmission failure during hypoxia (Hori and Carpenter, 1994). Our observations confirm that excitotoxic changes occur primarily at the postsynaptic dendrite (Olney, 1971). However, this does not exclude the possibility that hypoxic-ischemic injury (Stepanov et al., 1998) and other acute insults may also injure presynaptic terminals.

Resolution of neurological deficits after injury occurs through changes in the structure, function, or connectivity of surviving neurons. Rapid improvement after transmission failure may reflect restoration of ionic properties of presynaptic or postsynaptic neurons (Krnjevic, 1999); longer-term recovery of neuronal circuits may involve axonal sprouting, dendrite rearborization, or synaptogenesis (Kawamata et al., 1997; Nudo, 1999). In experimental animals, behavioral improvement after cortical or ischemic injury is associated with recovery of spine density (Kolb and Gibb, 1993; Akulinin et al., 1997). Rapid structural reconstitution of excitatory synapses is a possible substrate for recovery of function between neurons destined to survive excitotoxic or ischemic injury (Hasbani et al., 2000). This might occur in clinical settings of transient ischemic attack, brief cardiac arrest, or recovery from brain infarction in regions surrounding the ischemic core. Restoration of established synapses may be a novel target for therapeutic intervention to improve neurological function after acute brain injury.

\section{REFERENCES}

Akulinin VA, Stepanov SS, Semchenko VV, Belichenko PV (1997) Dendritic changes of the pyramidal neurons in layer $\mathrm{V}$ of sensory-motor cortex of the rat brain during the postresuscitation period. Resuscitation 35:157-164.

Allison DW, Gelfand VI, Spector I, Craig AM (1998) Role of actin in anchoring postsynaptic receptors in cultured hippocampal neurons: differential attachment of NMDA versus AMPA receptors. J Neurosci 18:2423-2436.

Crick F (1982) Do dendritic spines twitch? Trends Neurosci 5:44-46.

Dugan LL, Bruno VM, Amagasu SM, Giffard RG (1995) Glia modulate the response of murine cortical neurons to excitotoxicity: glia exacerbate AMPA neurotoxicity. J Neurosci 15:4545-4555.

Engert F, Bonhoeffer T (1999) Dendritic spine changes associated with hippocampal long-term synaptic plasticity. Nature 399:66-70.

Faddis BT, Hasbani MJ, Goldberg MP (1997) Calpain activation contributes to dendritic remodeling after brief excitotoxic injury in vitro. J Neurosci 17:951-959.

Fifkova E, Delay RJ (1982) Cytoplasmic actin in neuronal processes as a possible mediator of synaptic plasticity. J Cell Biol 95:345-350.

Fischer M, Kaech S, Knutti D, Matus A (1998) Rapid actin-based plasticity in dendritic spines. Neuron 20:847-854.

Goldberg MP, Choi DW (1993) Combined oxygen and glucose depriva- 
tion in cortical cell culture: calcium-dependent and calciumindependent mechanisms of neuronal injury. J Neurosci 13:3510-3524.

Goldberg MP, Strasser U, Dugan LL (1997) Techniques for assessing neuroprotective drugs in vitro. Int Rev Neurobiol 40:69-93.

Halpain S, Hipolito A, Saffer L (1998) Regulation of F-actin stability in dendritic spines by glutamate receptors and calcineurin. J Neurosci 18:9835-9844.

Harris KM, Kater SB (1994) Dendritic spines: cellular specializations imparting both stability and flexibility to synaptic function. Annu Rev Neurosci 17:341-371.

Hasbani MJ, Hyrc KL, Faddis BT, Romano C, Goldberg MP (1998) Distinct roles for sodium, chloride, and calcium in excitotoxic dendritic injury and recovery. Exp Neurol 154:241-258.

Hasbani MJ, Underhill SM, de Erausquin GA, Goldberg MP (2000) Synapse loss and regeneration: a mechanism for functional decline and recovery after cerebral ischemia? The Neuroscientist 6:110-119.

Honig MG, Hume RI (1986) Fluorescent carbocyanine dyes allow living neurons of identified origin to be studied in long-term cultures. J Cell Biol 103:171-187.

Hori N, Carpenter DO (1994) Functional and morphological changes induced by transient in vivo ischemia. Exp Neurol 129:279-289.

Hsu M, Buzsaki G (1993) Vulnerability of mossy fiber targets in the rat hippocampus to forebrain ischemia. J Neurosci 13:3964-3979.

Ikonomidou C, Price MT, Mosinger JL, Frierdich G, Labruyere J, Salles KS, Olney JW (1989) Hypobaric-ischemic conditions produce glutamate-like cytopathology in infant rat brain. J Neurosci 9:1693-1700.

Isokawa M (1998) Remodeling dendritic spines in the rat pilocarpine model of temporal lobe epilepsy. Neurosci Lett 258:73-76.

Jarvis CR, Lilge L, Vipond GJ, Andrew RD (1999) Interpretation of intrinsic optical signals and calcein fluorescence during acute excitotoxic insult in the hippocampal slice. NeuroImage 10:357-372.

Jiang M, Lee CL, Smith KL, Swann JW (1998) Spine loss and other persistent alterations of hippocampal pyramidal cell dendrites in a model of early-onset epilepsy. J Neurosci 18:8356-8368.

Kawamata T, Speliotes EK, Finklestein SP (1997) The role of polypeptide growth factors in recovery from stroke. Adv Neurol 73:377-382.

Kim CH, Lisman JE (1999) A role of actin filament in synaptic transmission and long-term potentiation. J Neurosci 19:4314-4324.

Kirov SA, Sorra KE, Harris KM (1999) Slices have more synapses than perfusion-fixed hippocampus from both young and mature rats. J Neurosci 19:2876-2886.

Kolb B, Gibb R (1993) Possible anatomical basis of recovery of function after neonatal frontal lesions in rats. Behav Neurosci 107:799-811.

Korkotian E, Segal M (1999a) Bidirectional regulation of dendritic spine dimensions by glutamate receptors. NeuroReport 10:2875-2877.

Korkotian E, Segal M (1999b) Release of calcium from stores alters the morphology of dendritic spines in cultured hippocampal neurons. Proc Natl Acad Sci USA 96:12068-12072.

Krnjevic K (1999) Early effects of hypoxia on brain cell function. Croat Med J 40:375-380.

Maletic-Savatic M, Malinow R, Svoboda K (1999) Rapid dendritic morphogenesis in CA1 hippocampal dendrites induced by synaptic activity. Science 283:1923-1927.

Matesic DF, Lin RC (1994) Microtubule-associated protein 2 as an early indicator of ischemia-induced neurodegeneration in the gerbil forebrain. J Neurochem 63:1012-1020.

Matus A (1999) Postsynaptic actin and neuronal plasticity. Curr Opin Neurobiol 9:561-565.

Muller M, Gahwiler BH, Rietschin L, Thompson SM (1993) Reversible loss of dendritic spines and altered excitability after chronic epilepsy in hippocampal slice cultures. Proc Natl Acad Sci USA 90:257-261.

Nudo RJ (1999) Recovery after damage to motor cortical areas. Curr Opin Neurobiol 9:740-747.

Okabe S, Kim HD, Miwa A, Kuriu T, Okado H (1999) Continual remodeling of postsynaptic density and its regulation by synaptic activity. Nat Neurosci 2:804-811.

Olney JW (1971) Glutamate-induced neuronal necrosis in the infant mouse hypothalamus. An electron microscopic study. J Neuropathol Exp Neurol 30:75-90.

Olney JW, Fuller T, de Gubareff T (1979) Acute dendrotoxic changes in the hippocampus of kainate treated rats. Brain Res 176:91-100.

Papa M, Bundman MC, Greenberger V, Segal M (1995) Morphological analysis of dendritic spine development in primary cultures of hippocampal neurons. J Neurosci 15:1-11.

Park JS, Bateman MC, Goldberg MP (1996) Rapid alterations in dendrite morphology during sublethal hypoxia or glutamate receptor activation. Neurobiol Dis 3:215-227.

Ramon y Cajal S (1909) Histologie du systeme nerveux de l'homme et des vertebres. Paris: Maloine.

Ramon y Cajal S (1995) Histology of the nervous system. Oxford: Oxford UP.

Rose K, Goldberg MP, Choi DW (1993) Cytotoxicity in murine cortical cell culture. In: In vitro biological methods. Methods in toxicology (Tyson CA, Frazier JM, eds), pp 46-60. San Diego: Academic.

Rosenmund C, Westbrook GL (1993) Calcium-induced actin depolymerization reduces NMDA channel activity. Neuron 10:805-814.

Rowntree S, Kolb B (1997) Blockade of basic fibroblast growth factor retards recovery from motor cortex injury in rats. Eur J Neurosci 9:2432-2441.

Sattler R, Xiong Z, Lu WY, MacDonald JF, Tymianski M (2000) Distinct roles of synaptic and extrasynaptic NMDA receptors in excitotoxicity. J Neurosci 20:22-33.

Segal M (1995) Dendritic spines for neuroprotection: a hypothesis. Trends Neurosci 18:468-471.

Segal M, Korkotian E, Murphy DD (2000) Dendritic spine formation and pruning: common cellular mechanisms? Trends Neurosci 23:53-57.

Stepanov SS, Sergeyeva ED, Semchenko VV, Akulinin VA (1998) An ultrastructural study into the effect of global transient cerebral ischaemia on the synaptic population of the cerebellar cortex in rats. Resuscitation 39:99-106.

Stewart GR, Olney JW, Pathikonda M, Snider WD (1991) Excitotoxicity in the embryonic chick spinal cord. Ann Neurol 30:758-766.

Toni N, Buchs PA, Nikonenko I, Bron CR, Muller D (1999) LTP promotes formation of multiple spine synapses between a single axon terminal and a dendrite. Nature 402:421-425.

Yin Hz, Turetsky D, Choi DW, Weiss JH (1994) Cortical neurons with $\mathrm{Ca} 2+$ permeable AMPA/kainate channels display distinct receptor immunoreactivity and are GABAergic. Neurobiol Dis 1:43-49.

Ziv NE, Smith SJ (1996) Evidence for a role of dendritic filopodia in synaptogenesis and spine formation. Neuron 17:91-102. 